\title{
On Quadrirational Yang-Baxter Maps ${ }^{\star}$
}

\author{
V.G. PAPAGEORGIOU $\dagger^{\dagger^{1}}$, Yu.B. SURIS $\dagger^{2}$, A.G. TONGAS $\dagger^{\dagger^{3}}$ and A.P.VESELOV ${ }^{\dagger^{4} \dagger^{5}}$ \\ $\dagger^{1}$ Department of Mathematics, University of Patras, 26500 Patras, Greece \\ E-mail: vassilis@math.upatras.gr \\ $\dagger^{2}$ Institut für Mathematik, Technische Universität Berlin, \\ Str. des 17. Juni 136, 10623 Berlin, Germany \\ E-mail: suris@math.tu-berlin.de \\ $\dagger^{3}$ Department of Applied Mathematics, University of Crete, 71409 Heraklion, Greece \\ E-mail: atongas@tem.uoc.gr \\ $\dagger^{4}$ School of Mathematics, Loughborough University, \\ Loughborough, Leicestershire, LE11 3TU, UK \\ E-mail: A.P.Veselov@lboro.ac.uk \\ $\dagger^{5}$ Moscow State University, Moscow 119899, Russia
}

Received November 15, 2009, in final form March 26, 2010; Published online April 16, 2010

doi:10.3842/SIGMA.2010.033

\begin{abstract}
We use the classification of the quadrirational maps given by Adler, Bobenko and Suris to describe when such maps satisfy the Yang-Baxter relation. We show that the corresponding maps can be characterized by certain singularity invariance condition. This leads to some new families of Yang-Baxter maps corresponding to the geometric symmetries of pencils of quadrics.
\end{abstract}

Key words: Yang-Baxter maps; birational maps; integrability

2010 Mathematics Subject Classification: 14E07; 14H70; 37K20

\section{Introduction}

Recently Adler, Bobenko and Suris [1] introduced an important notion of quadrirational maps of $\mathbb{C P}^{1} \times \mathbb{C P}^{1}$ into itself. They classified them up to a natural $(\mathcal{M} \ddot{o} b)^{4}$-action, where $\mathcal{M o ̈ b}$ is the Möbius group of the projective transformations of $\mathbb{C P}^{1}$, and gave a beautiful geometric interpretation of the results.

In this note, which can be considered as an extended comment to [1], we use the classification of the quadrirational maps from [1] to describe when such maps satisfy the Yang-Baxter relation. We show that the corresponding maps can be characterized by certain singularity invariance condition, which leads to the 5 families of Yang-Baxter maps found in [1], as well as to 5 additional families corresponding to the geometric symmetries of pencils of quadrics.

We should say that a surprising relation of the quadrirationality with the Yang-Baxter property was discovered already in [1]. However it was left without proper discussion, which may lead to misinterpretation of the main result (see the concluding remarks in [8]). We came naturally to this point when new examples of quadrirational Yang-Baxter maps were discovered in $[5]$.

${ }^{\star}$ This paper is a contribution to the Proceedings of the Workshop "Geometric Aspects of Discrete and UltraDiscrete Integrable Systems" (March 30 - April 3, 2009, University of Glasgow, UK). The full collection is available at http://www.emis.de/journals/SIGMA/GADUDIS2009.html 


\section{Yang-Baxter relation and quadrirational maps}

Let $\mathbb{X}$ be any set and $R$ a map of $\mathbb{X} \times \mathbb{X}$ into itself. If $\mathbb{X}^{n}$ stands for $\mathbb{X} \times \mathbb{X} \times \cdots \times \mathbb{X}$, then let $R^{i j}$ will denote the map of $\mathbb{X}^{n}$ into itself which acts as $R$ on the $i$-th and $j$-th factors and as the identity on the others. A map $R(\lambda, \mu): \mathbb{X} \times \mathbb{X} \rightarrow \mathbb{X} \times \mathbb{X}$ depending on two parameters $\lambda, \mu$ from some parameter set $\Lambda$ is called a Yang-Baxter map if it satisfies the Yang-Baxter relation

$$
R^{23}\left(\lambda_{2}, \lambda_{3}\right) R^{13}\left(\lambda_{1}, \lambda_{3}\right) R^{12}\left(\lambda_{1}, \lambda_{2}\right)=R^{12}\left(\lambda_{1}, \lambda_{2}\right) R^{13}\left(\lambda_{1}, \lambda_{3}\right) R^{23}\left(\lambda_{2}, \lambda_{3}\right),
$$

regarded as an equality of maps of $\mathbb{X} \times \mathbb{X} \times \mathbb{X}$ into itself. If in addition the relation

$$
R^{21}\left(\lambda_{2}, \lambda_{1}\right) R\left(\lambda_{1}, \lambda_{2}\right)=\mathrm{Id}
$$

holds, then $R(\lambda, \mu)$ is called a reversible Yang-Baxter map.

The following proposition, which is easily checked directly, defines a natural equivalence among the parameter-dependent Yang-Baxter maps.

Proposition 1. Assume that there is a family of bijections $\phi(\lambda): \mathbb{X} \rightarrow \mathbb{X}$ parametrized by $\lambda \in \Lambda$. If $R(\lambda, \mu)$ satisfies the Yang-Baxter relation (1), then the same is true for

$$
\widetilde{R}(\lambda, \mu)=\phi(\lambda)^{-1} \times \phi(\mu)^{-1} R(\lambda, \mu) \phi(\lambda) \times \phi(\mu) .
$$

The Yang-Baxter maps $R, \widetilde{R}$ are called equivalent.

Parameter dependent Yang-Baxter maps can be considered as parameter independent by extending the domain $\mathbb{X}$ to $\mathbb{X} \times \Lambda$ with identical action of the map on the second factor. However this may change the meaning of natural equivalence between the maps.

From now on we will be mainly interested in the case $\mathbb{X}=\mathbb{C P}^{1}$.

Recall that a map of $\mathbb{C P}^{1} \times \mathbb{C P}^{1}$ into itself, $R:(x, y) \mapsto(u, v)$, is called quadrirational [1] if both $R$ and the so-called companion map $\bar{R}:(x, v) \mapsto(u, y)$ are birational maps. All such maps have the form

$$
R: u=\frac{a(y) x+b(y)}{c(y) x+d(y)}, \quad v=\frac{A(x) y+B(x)}{C(x) y+D(x)},
$$

where $a(y), \ldots, d(y)$ and $A(x), \ldots, D(x)$ are polynomials of degree at most 2 . There are three subclasses of such maps, denoted as [1:1], [1:2] and [2:2], corresponding to the highest degrees of the coefficients of both fractions in (3).

In this paper we restrict ourselves by the most interesting subclass [2:2]. Adler, Bobenko and Suris [1] showed that any quadrirational map from this subclass is $(\mathcal{M} \ddot{o} b)^{4}$-equivalent to one of the following five maps depending on two complex parameters $\alpha, \beta$ :

$$
\begin{aligned}
& u=\alpha y P, \quad v=\beta x P, \quad P=\frac{(1-\beta) x+\beta-\alpha+(\alpha-1) y}{\beta(1-\alpha) x+(\alpha-\beta) y x+\alpha(\beta-1) y}, \\
& u=\frac{y}{\alpha} P, \quad v=\frac{x}{\beta} P, \quad P=\frac{\alpha x-\beta y+\beta-\alpha}{x-y}, \\
& u=\frac{y}{\alpha} P, \quad v=\frac{x}{\beta} P, \quad P=\frac{\alpha x-\beta y}{x-y}, \\
& u=y P, \quad v=x P, \quad P=1+\frac{\beta-\alpha}{x-y}, \\
& u=y+P, \quad v=x+P, \quad P=\frac{\alpha-\beta}{x-y} \text {. }
\end{aligned}
$$


A surprising fact is that all five canonical representative maps satisfy the Yang-Baxter relation

$$
R^{23}\left(\alpha_{2}, \alpha_{3}\right) R^{13}\left(\alpha_{1}, \alpha_{3}\right) R^{12}\left(\alpha_{1}, \alpha_{2}\right)=R^{12}\left(\alpha_{1}, \alpha_{2}\right) R^{13}\left(\alpha_{1}, \alpha_{3}\right) R^{23}\left(\alpha_{2}, \alpha_{3}\right) .
$$

However, it should be noted that not all quadrirational maps satisfy the Yang-Baxter relation (as one might conclude from [1]), since the action of $(\mathcal{M} \ddot{o} b)^{4}$, in general, destroys the YangBaxter property. For example, if we change in $F_{\mathrm{V}}$ the variables $x, y, u, v$ to $-x,-y, u$, $v$, we come to the map

$$
u=-(y+P), \quad v=-(x+P), \quad P=\frac{\alpha-\beta}{x-y},
$$

which does not satisfy the Yang-Baxter property (see [8]).

\section{Yang-Baxter property and singularity invariance}

To formulate a criterium for a quadrirational map to satisfy the Yang-Baxter relation, we start with the parametrization of the quadrirational maps in terms of their singularity sets following Adler-Bobenko-Suris [1]. For simplicity we restrict our considerations to the generic case corresponding to the $(\mathcal{M} \ddot{o} b)^{4}$-orbits of the maps of type $F_{\mathrm{I}}$. In that case the corresponding quadrirational map $F$ has the singular set $\Sigma(F)=\left\{P_{1}, \ldots, P_{4}\right\}$ consisting of four distinct points $P_{i}=\left(x_{i}, y_{i}\right) \in \mathbb{C P}^{1} \times \mathbb{C P}^{1}$ (where the numerators and the denominators of the both fractions in (3) simultaneously vanish). Analogously, the map $F^{-1}$ has the singular set $\Sigma\left(F^{-1}\right)=$ $\left\{Q_{1}, \ldots, Q_{4}\right\}$ consisting of four distinct points $Q_{i}=\left(u_{i}, v_{i}\right) \in \mathbb{C P}^{1} \times \mathbb{C P}^{1}$. Moreover, each of the companion maps $\bar{R}$ and $\bar{R}^{-1}$ has four distinct singular points, given by $\left(x_{i}, v_{i}\right)$ and $\left(u_{i}, y_{i}\right)$, respectively. It can be shown that $F$ blows up the points $P_{i}$ and blows down four exceptional curves $C_{i}$ of bidegree $(1,1)$ to the points $Q_{i}$. The exceptional curves can be ordered in such a way that $C_{i}$ passes through three points $P_{j}, j \neq i$ (see Theorems 21 and 22 in [1]).

Note that an ordered set $X=\left(x_{1}, \ldots, x_{4}\right)$ of 4 distinct points in $\mathbb{C P}^{1}$ is in a one-to-one correspondence with the set of pairs $(\alpha, \sigma), \alpha \in \mathbb{C}, \sigma \in \mathcal{M} \ddot{o} b$. Indeed, $\alpha$ is defined as the cross ratio

$$
\alpha=q(X)=\frac{\left(x_{1}-x_{2}\right)\left(x_{3}-x_{4}\right)}{\left(x_{2}-x_{3}\right)\left(x_{4}-x_{1}\right)}
$$

and $\sigma$ is uniquely defined by the condition that

$$
\sigma\left(x_{1}\right)=\infty, \quad \sigma\left(x_{2}\right)=1, \quad \sigma\left(x_{3}\right)=0, \quad \sigma\left(x_{4}\right)=\alpha,
$$

giving

$$
\sigma(x)=\frac{\left(x_{1}-x_{2}\right)\left(x-x_{3}\right)}{\left(x_{3}-x_{2}\right)\left(x-x_{1}\right)} .
$$

Let $X=\left(x_{1}, \ldots, x_{4}\right), Y=\left(y_{1}, \ldots, y_{4}\right), U=\left(u_{1}, \ldots, u_{4}\right), V=\left(v_{1}, \ldots, v_{4}\right)$ be four ordered quadruples of elements of $\mathbb{C P}^{1}$. According to Theorem 18 in [1], they correspond to some quadrirational map if and only if $U=\sigma(X), V=\tau(Y)$ for some $\sigma, \tau \in \mathcal{M} \ddot{o} b$, that is, if $q(X)=q(U)$ and $q(Y)=q(V)$. We denote the corresponding map by $F(\lambda, \mu)$, where $\lambda=(X, U)$, $\mu=(Y, V)$ belong to the admissible set

$$
\Lambda=\left\{(X, U) \in\left(\mathbb{C P}^{1}\right)^{4} \times\left(\mathbb{C P}^{1}\right)^{4}: U=\sigma(X) \text { with } \sigma \in \mathcal{M} \ddot{b} b .\right.
$$

Clearly, a simultaneous permutation of all four quadruples $X, Y, U, V$ leads to the same map $F$. 
We now turn to the Yang-Baxter relation for the class of quadrirational maps. In the formulation of our main result, we use the following notation. Let $\pi \in S_{4}$ be a permutation of indices $(1, \ldots, 4)$. Then

$$
\Lambda_{\pi}=\left\{(X, U) \in \Lambda: U=X^{\pi} \Leftrightarrow u_{i}=x_{\pi(i)}, i=1, \ldots, 4\right\} .
$$

Since in this definition $U$ must be $\mathcal{M} \ddot{o} b$-equivalent to $X$, the permutation $\pi$ must preserve the cross ratio and thus belongs to the Klein subgroup $K \subset S_{4}$ consisting of the identity Id and the three commuting involutions

$$
\rho_{1}=(12)(34), \quad \rho_{2}=(14)(23), \quad \rho_{3}=(13)(24) .
$$

Note that any two of $\rho_{i}$ are conjugated in $S_{4}$.

Theorem 1. The map $F(\lambda, \mu)$ satisfies the Yang-Baxter relation (1) if and only if all parameters $\lambda_{1}, \lambda_{2}, \lambda_{3}$ belong to the same subset $\Lambda_{\pi} \subset \Lambda, \pi \in K$.

Note that for $F(\lambda, \mu)$ with $\lambda, \mu \in \Lambda_{\pi}$ the singular set of the inverse map consists of $\left(u_{i}, v_{i}\right)=$ $\left(x_{\pi_{i}}, y_{\pi_{i}}\right)$, and coincides with the singular set of $F$, which can be considered as the following version of the singularity confinement condition.

Let us say that a quadrirational map $F$ satisfies singularity invariance ${ }^{1}$ property if the singular set of $F^{-1}$ coincides with that of the map $F$. We see that the condition $\lambda, \mu \in \Lambda_{\pi}$ is precisely the singularity invariance for the corresponding map $F(\lambda, \mu)$.

Thus, our main observation is that the Yang-Baxter property for the quadrirational maps is equivalent to the singularity invariance. In particular, this explains why the canonical maps $F_{\mathrm{I}}-F_{\mathrm{V}}$ satisfy the Yang-Baxter relation. For example, for the map $F_{\mathrm{I}}$ we have:

$$
X=U=(\infty, 1,0, \alpha), \quad Y=V=(\infty, 1,0, \beta),
$$

so that the singularity set is

$$
\Sigma\left(F_{\mathrm{I}}\right)=\{(\infty, \infty),(1,1),(0,0),(\alpha, \beta)\}=\Sigma\left(F_{\mathrm{I}}^{-1}\right) .
$$

A quadrirational map $R$ with confined singularities determines a permutation $\pi(R)$ of the singularity set $\Sigma(R)$. In the $F_{\mathrm{I}}$ case this permutation is identity, but this need not be the case in general.

For example, if we change in $F_{\mathrm{I}}$ the variables $y \mapsto \beta y^{-1}, u \mapsto \alpha u^{-1}$, while keeping $x, v$ unchanged, we obtain a non-equivalent map

$$
u=y Q^{-1}, \quad v=x Q, \quad Q=\frac{(1-\beta) x y+(\beta-\alpha) y+\beta(\alpha-1)}{(1-\alpha) x y+(\alpha-\beta) x+\alpha(\beta-1)},
$$

which we denote by $H_{\mathrm{I}}$. For this map we have:

$$
X=(\infty, 1,0, \alpha), \quad Y=(0, \beta, \infty, 1), \quad U=(0, \alpha, \infty, 1), \quad V=(\infty, 1,0, \beta) .
$$

The singularity set is

$$
\Sigma\left(H_{\mathrm{I}}\right)=\{(\infty, 0),(1, \beta),(0, \infty),(\alpha, 1)\}=\Sigma\left(H_{\mathrm{I}}^{-1}\right),
$$

and the corresponding permutation is $\pi=\rho_{3}=(13)(24)$. Thus, the map $H_{\mathrm{I}}$ has the singularity invariance property and, correspondingly, it satisfies the Yang-Baxter relation. It is conjugated to the so-called Harrison map found in [5], see also [3,6]. Similar maps can be constructed

\footnotetext{
${ }^{1}$ A closely related property called by Duistermaat [2] geometric confinement was introduced in [9].
} 
for other canonical maps except $F_{\mathrm{IV}}$ (see the full list in Section 4 below). In the following we elucidate these issues in detail.

To prove the theorem recall that according to Theorem 19 in [1], multidimensionally consistent systems of quadrirational maps are characterized by matching of singularities in the following way. (We restrict ourselves here again with the generic case of the systems consisting of maps from the $(\mathcal{M} \ddot{o} b)^{4}$-orbit of $F_{\mathrm{I}}$.) Let each edge of the three-dimensional cube carry an ordered quadruple of elements of $\mathbb{C P}^{1}$, such that the cross-ratios of the quadruples on the opposite edges of each face coincide. We denote these quadruples by the the same letters as the corresponding fields on Fig. 1, but use the capital case. Attach to each face of the cube a quadrirational map according to this data. Then this system of maps is three-dimensionally consistent.

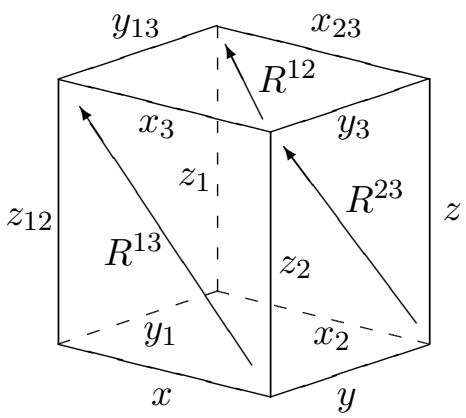

Figure 1. Right-hand side of Yang-Baxter equation; the left hand side is the composition of the maps corresponding to the other three faces of the cube.

In order for the maps on the bottom and on the top faces to coincide it is necessary and sufficient that the corresponding eight quadruples be related by

$$
\left(X_{3}, Y_{3}, X_{23}, Y_{13}\right)=\left(X, Y, X_{2}, Y_{1}\right)^{\pi_{3}}
$$

with some permutation $\pi_{3} \in S_{4}$. (This is to be read as the simultaneous permutation of all four quadruples, so that $X_{3}=X^{\pi_{3}}$ etc.) Equal cross-ratios for opposite edges implies, as discussed above, that $\pi_{3} \in K$; in particular, it is an involution. Similarly, each of the other two pairs consists of the same maps if

$$
\left(Y_{1}, Z_{1}, Y_{13}, Z_{12}\right)=\left(Y, Z, Y_{3}, Z_{2}\right)^{\pi_{1}}, \quad\left(X, Z_{2}, X_{3}, Z_{12}\right)=\left(X_{2}, Z, X_{23}, Z_{1}\right)^{\pi_{2}}
$$

with some involutive permutations $\pi_{1}, \pi_{2} \in K \subset S_{4}$. These relations are equivalent to the following ones: first,

$$
\begin{array}{ll}
\left(X, X_{2}\right) \in \Lambda_{\pi_{2}}, & \left(Y, Y_{1}\right) \in \Lambda_{\pi_{1}}, \\
\left(Y, Y_{3}\right) \in \Lambda_{\pi_{3}}, & \left(Z, Z_{2}\right) \in \Lambda_{\pi_{2}}, \\
\left(X, X_{3}\right) \in \Lambda_{\pi_{3}}, & \left(Z, Z_{1}\right) \in \Lambda_{\pi_{1}},
\end{array}
$$

and second, that any two of the permutations $\pi_{1}, \pi_{2}, \pi_{3}$ commute. In order for the corresponding maps to be identified with $F^{12}\left(\lambda_{1}, \lambda_{2}\right), F^{23}\left(\lambda_{2}, \lambda_{3}\right)$, and $F^{13}\left(\lambda_{1}, \lambda_{3}\right)$, it is required that $\lambda_{1}=$ $\left(X, X_{2}\right)=\left(X, X_{3}\right), \lambda_{2}=\left(Y, Y_{1}\right)=\left(Y, Y_{3}\right)$, and $\lambda_{3}=\left(Z, Z_{1}\right)=\left(Z, Z_{2}\right)$. Therefore, we arrive at the condition $\pi_{1}=\pi_{2}=\pi_{3}$, which proves the theorem.

When $\pi=(13)(24)$ we arrive at the map $H_{\mathrm{I}}$ defined above. For other nontrivial elements of the Klein group we have different families of maps, which however turn out to be equivalent as families if we allow also change of parameters (see below). This corresponds to the fact that the nontrivial elements of the Klein subgroup are conjugated to one another. 
Thus, in the generic case we have two non-equivalent 8-parameter families of quadrirational Yang-Baxter maps:

$$
\begin{array}{ll}
R(X, Y)=F((X, X),(Y, Y))=\sigma^{-1} \times \tau^{-1} F_{\mathrm{I}}(\alpha, \beta) \sigma \times \tau & \left(F_{\mathrm{I}} \text {-family }\right), \\
R(X, Y)=F\left(\left(X, X^{\rho}\right),\left(Y, Y^{\rho}\right)\right)=\sigma^{-1} \times \tau^{-1} H_{\mathrm{I}}(\alpha, \beta) \sigma \times \tau & \left(H_{\mathrm{I}} \text {-family }\right),
\end{array}
$$

where $\rho=(13)(24), X, Y \in\left(\mathbb{C P}^{1}\right)^{4}$ are ordered sets of 4 distinct points in $\mathbb{C P}^{1}$ and $\sigma, \tau \in \mathcal{M} \ddot{b}$.

Of course these maps are equivalent, in the sense of Proposition 1 , to the maps $F_{\mathrm{I}}$ and $H_{\mathrm{I}}$ respectively. Next, we are going to show that additional families correspond to the symmetries of the pencils of quadrics.

\section{Geometric symmetries and additional families of Yang-Baxter maps}

The relation between the maps $F_{\mathrm{I}}$ and $H_{\mathrm{I}}$ can be also established with the help of the following statement. We say that a family of involutions $\sigma(\lambda)$ of the set $\mathbb{X}$ is a symmetry of a reversible YB map $R(\lambda, \mu): \mathbb{X} \times \mathbb{X} \rightarrow \mathbb{X} \times \mathbb{X}$ if

$$
\sigma(\lambda) \times \sigma(\mu) R(\lambda, \mu)=R(\lambda, \mu) \sigma(\lambda) \times \sigma(\mu) .
$$

The following simple but important statement is easily proved by a direct check.

Proposition 2. If $\sigma(\lambda)$ is a symmetry of a reversible $Y B$ map $R(\lambda, \mu)$, then

$$
R^{\sigma}=\sigma(\lambda) \times \operatorname{Id} R(\lambda, \mu) \operatorname{Id} \times \sigma(\mu)
$$

is also a reversible YB map.

A nice way to find symmetries of the canonical maps $F_{\mathrm{I}}-F_{\mathrm{V}}$ is provided by their geometric interpretation given in [1] and illustrated on Fig. 2. Consider two conics $Q_{1}, Q_{2}$ on the plane and let $\mathbf{X} \in Q_{1}, \mathbf{Y} \in Q_{2}$. Set $\mathbf{U}(\mathbf{V})$ to be the second intersection point of the line $\overline{\mathbf{X Y}}$ with $Q_{1}$ (resp. with $\left.Q_{2}\right)$. Then the maps $F_{\mathrm{I}}-F_{\mathrm{V}}:(x, y) \mapsto(u, v)$ are just the coordinate representations of the map $\mathcal{R}:(\mathbf{X}, \mathbf{Y}) \mapsto(\mathbf{U}, \mathbf{V})$ in some special rational parametrization of the conics, $\alpha$ and $\beta$ being the cross-ratios of the corresponding four intersection points. The five maps $F_{\mathrm{I}}-F_{\mathrm{V}}$ correspond to the five possible types $\mathrm{I}-\mathrm{V}$ of intersection of two conics:

I: four simple intersection points;

II: two simple intersection points and one point of tangency;

III: two points of tangency;

IV: one simple intersection point and one point of the second order tangency;

$\mathrm{V}$ : one point of the third order tangency.

Let us examine first the symmetries of the $F_{\mathrm{I}}$ map. The pair of quadrics in the $w_{1}-w_{2}$ plane, in generic position, can be reduced by a suitable projective transformation to

$$
Q_{1}: w_{2}\left(w_{2}-1\right)=\alpha w_{1}\left(w_{1}-1\right), \quad Q_{2}: w_{2}\left(w_{2}-1\right)=\beta w_{1}\left(w_{1}-1\right) .
$$

It has an obvious symmetry group $K=\mathbb{Z}_{2} \times \mathbb{Z}_{2}=\left\{\mathrm{Id}, \rho_{1}, \rho_{2}, \rho_{3}\right\}$, where

$$
\rho_{1}\left(w_{1}, w_{2}\right)=\left(1-w_{1}, w_{2}\right), \quad \rho_{2}\left(w_{1}, w_{2}\right)=\left(w_{1}, 1-w_{2}\right), \quad \rho_{3}\left(w_{1}, w_{2}\right)=\left(1-w_{1}, 1-w_{2}\right)
$$

(see Fig. 3). 


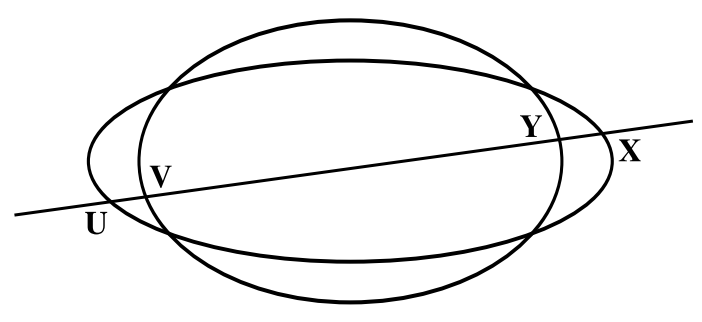

Figure 2. A quadrirational map on a pair of conics.

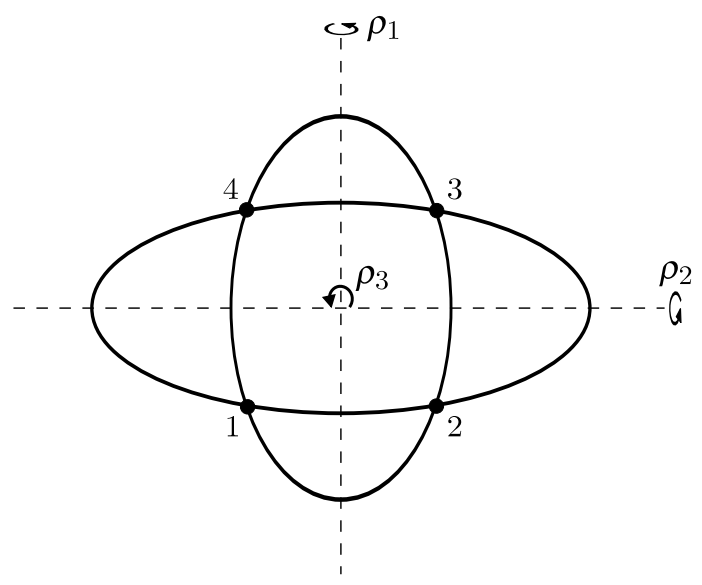

Figure 3. Involutions of the pair of quadrics in the generic case.

In the parametrization

$$
w_{1}=\frac{x-\alpha}{x^{2}-\alpha}, \quad w_{2}=\frac{x(x-\alpha)}{x^{2}-\alpha}
$$

for the first quadric and similarly for the second one with $\alpha$ replaced by $\beta$, the symmetry $\rho_{3}$ corresponds to

$$
\sigma(\alpha): x \mapsto \frac{\alpha}{x}
$$

By applying Proposition 2 to the map $F_{\mathrm{I}}$ with this symmetry, we obtain the map $H_{\mathrm{I}}$ (see Fig. 4).

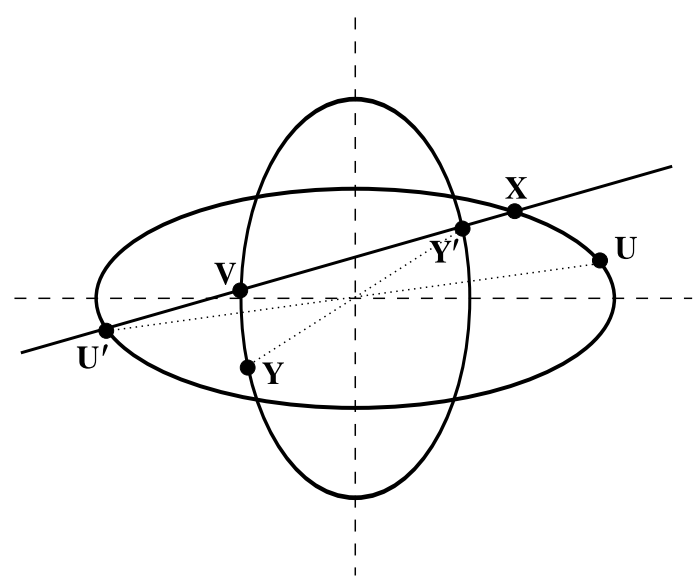

Figure 4. Geometric interpretation of $H_{I}$ map. 
Application of Proposition 2 to $F_{\mathrm{I}}$ with the symmetry $\rho_{1}$, which corresponds to

$$
\tau(\alpha): x \mapsto \frac{x-\alpha}{x-1},
$$

leads to the YB map

$$
u=\frac{\alpha y(\beta-x-y+x y)}{\alpha \beta-\beta x-\alpha y+\beta x y}, \quad v=\frac{\beta x(\alpha-x-y+x y)}{\alpha \beta-\beta x-\alpha y+\alpha x y} .
$$

It is, however, equivalent to the map $H_{\mathrm{I}}$ via the conjugation by $x \mapsto(x-1) / x$ and the reparametrization $\alpha \mapsto(\alpha-1) / \alpha$. Similarly, the third symmetry $\rho_{2}$ leads to the family which is related to the map (4) by conjugation $x \mapsto 1 / x$ and reparametrization $\alpha \mapsto 1 / \alpha$.

In the degenerate cases $F_{\mathrm{II}}$ and $F_{\mathrm{V}}$ the symmetry group breaks down to $\mathbb{Z}_{2}$, so we have one additional family for each case. In case $F_{\text {III }}$ we can choose the equations of quadrics as

$$
Q_{1}: \alpha w_{1}\left(w_{1}-1\right)=w_{2}^{2}, \quad Q_{2}: \beta w_{1}\left(w_{1}-1\right)=w_{2}^{2},
$$

and we still have $\mathbb{Z}_{2} \times \mathbb{Z}_{2}$ symmetry group. One can check that the symmetries $\left(w_{1}, w_{2}\right) \mapsto$ $\left(1-w_{1}, \pm w_{2}\right)$ lead to equivalent families, so we have two new families in this case, depending whether the points of tangency are interchanged ( $B$-family) or not ( $A$-family). In the case $F_{\mathrm{IV}}$ we have no non-trivial symmetries. This leads to the following list.

Theorem 2. Up to equivalence (2) and reparametrization there are 10 families of quadrirational reversible Yang-Baxter maps of subclass [2:2]: the 5 families $F_{\mathrm{I}}-F_{\mathrm{V}}$ from [1] and the following 5 additional families

$$
\begin{aligned}
& u=y Q^{-1}, \quad v=x Q, \quad Q=\frac{(1-\beta) x y+(\beta-\alpha) y+\beta(\alpha-1)}{(1-\alpha) x y+(\alpha-\beta) x+\alpha(\beta-1)}, \\
& u=y Q^{-1}, \quad v=x Q, \quad Q=\frac{\alpha+(\beta-\alpha) y-\beta x y}{\beta+(\alpha-\beta) x-\alpha x y}, \\
& u=\frac{y}{\alpha} Q, \quad v=\frac{x}{\beta} Q, \quad Q=\frac{\alpha x+\beta y}{x+y}, \\
& u=y Q^{-1}, \quad v=x Q, \quad Q=\frac{\alpha x y+1}{\beta x y+1}, \\
& u=y-P, \quad v=x+P, \quad P=\frac{\alpha-\beta}{x+y} .
\end{aligned}
$$

The map $H_{\mathrm{V}}$ is the well-known Adler map (see [7]). The map $H_{\mathrm{III}}^{B}$ appeared in [5] in relation with the discrete potential $\mathrm{KdV}$ equation and in [4] in relation with the discrete KP hierarchy.

Note that $H_{\mathrm{III}}^{A}$ and $H_{\mathrm{V}}$ can be obtained from $F_{\mathrm{III}}$ and $F_{\mathrm{V}}$, respectively, by the change $u \mapsto-u$, $y \mapsto-y$.

The maps $H_{\mathrm{I}}, H_{\mathrm{II}}$ have convenient subtraction-free representatives:

$$
\begin{aligned}
u & =\frac{y}{A} \frac{B+A x+B y+A B x y}{1+x+y+B x y}, & v & =\frac{x}{B} \frac{A+A x+B y+A B x y}{1+x+y+A x y}, \\
u & =\frac{y}{\alpha} \frac{\alpha x+\beta y+\beta}{x+y+1}, & v & =\frac{x}{\beta} \frac{\alpha x+\beta y+\alpha}{x+y+1},
\end{aligned}
$$

where $A=1-\alpha, B=1-\beta$. They are related to the maps $H_{\mathrm{I}}, H_{\mathrm{II}}$ above by conjugation viz. $x \mapsto 1 /(x-1)$ and similarly for all other variables. Note that the maps $H_{\mathrm{III}}^{A}$ and $H_{\mathrm{III}}^{B}$ are subtraction-free also, so $H_{\mathrm{I}}^{+}, H_{\mathrm{II}}^{+}, H_{\mathrm{III}}^{A}$ and $H_{\mathrm{III}}^{B}$ have a natural "tropical" (or ultra-discrete) limit (cf. [4]). Moreover there is an obvious (singular) limit procedure starting from $H_{\mathrm{I}}^{+}$in order to obtain $H_{\mathrm{II}}^{+}$and $H_{\mathrm{III}}^{A}$. 
Although the families of maps $F$ and $H$ are not equivalent it is not clear if they lead to different transfer dynamics [7]. For example, both $F_{\mathrm{V}}$ and $H_{\mathrm{V}}$ are related to the discrete (potential) KdV equation, but via different symmetries of it (see [5]). However, we know that considering other symmetries of discrete $\mathrm{KdV}$ equation one can obtain the families $F_{\mathrm{IV}}$ and even $H_{\mathrm{I}}($ see $[5])$.

Note also that although all the maps $F_{\mathrm{I}}-F_{\mathrm{V}}, H_{\mathrm{I}}-H_{\mathrm{V}}$ are the involutions, the corresponding transfer-dynamics [7] is non-trivial and deserves further investigation.

\section{Acknowledgements}

This work had been started in May 2007, when one of the authors (APV) visited Patras within the Erasmus-Socrates exchange programme, and completed at the Isaac Newton Institute for Mathematical Sciences in Cambridge during the programme on Discrete Integrable Systems in the spring semester 2009. The work of APV was also partially supported by the European RTN ENIGMA (contract MRTN-CT-2004-5652) and EPSRC (grant EP/E004008/1). We are grateful to V. Adler for useful comments.

\section{References}

[1] Adler V.E., Bobenko A.I., Suris Yu.B., Geometry of Yang-Baxter maps: pencils of conics and quadrirational mappings, Comm. Anal. Geom. 12 (2004), 967-1007, math.QA/0307009.

[2] Duistermaat J.J., QRT and elliptic surfaces, Springer, Universitext, 2010.

[3] Harrison B.K., Bäcklund transformation for the Ernst equation of general relativity, Phys. Rev. Lett. 41 (1978), 1197-1200.

[4] Kakei S., Nimmo J.J.C., Willox R., Yang-Baxter maps and the discrete KP hierarchy, Glasg. Math. J. 51 (2009), no. A, 107-119.

[5] Papageorgiou V.G., Tongas A.G., Veselov A.P., Yang-Baxter maps and symmetries of integrable equations on quad-graphs, J. Math. Phys. 47 (2006), 083502, 16 pages, math.QA/0605206.

[6] Tongas A., Tsoubelis D., Xenitidis P., A family of integrable nonlinear equations of hyperbolic type, J. Math. Phys. 42 (2001), 5762-5784.

[7] Veselov A.P., Yang-Baxter maps and integrable dynamics, Phys. Lett. A 314 (2003), 214-221, math.QA/0205335.

[8] Veselov A.P., Yang-Baxter maps: dynamical point of view, in Combinatorial Aspect of Integrable Systems, MSJ Mem., Vol. 17, Math. Soc. Japan, Tokyo, 2007, 145-167, math.QA/0612814.

[9] Veselov A.P., Integrable mappings, Russian Math. Surveys 46 (1991), no. 5, 1-51. 